\title{
A QUANTITATIVE REVERSE FABER-KRAHN INEQUALITY FOR THE FIRST ROBIN EIGENVALUE WITH NEGATIVE BOUNDARY PARAMETER
}

\author{
Simone Cito $^{1, *}$ And Domenico Angelo La Manna ${ }^{2}$
}

\begin{abstract}
The aim of this paper is to prove a quantitative form of a reverse Faber-Krahn type inequality for the first Robin Laplacian eigenvalue $\lambda_{\beta}$ with negative boundary parameter among convex sets of prescribed perimeter. In that framework, the ball is the only maximizer for $\lambda_{\beta}$ and the distance from the optimal set is considered in terms of Hausdorff distance. The key point of our stategy is to prove a quantitative reverse Faber-Krahn inequality for the first eigenvalue of a Steklov-type problem related to the original Robin problem.
\end{abstract}

Mathematics Subject Classification. 35P15, 35B35, 49Q10, 49R05.

Received May 26, 2020. Accepted November 12, 2020.

\section{INTRODUCTION}

Let $\Omega \subset \mathbb{R}^{n}$ be a bounded Lipschitz domain and $\beta>0$. A number $\lambda \in \mathbb{R}$ is an eigenvalue of the RobinLaplacian with boundary parameter $-\beta$ in $\Omega$ if there exists a non-zero function $u \in H^{1}(\Omega)$ solving the problem

$$
\begin{cases}-\Delta u=\lambda u & \text { in } \Omega \\ \frac{\partial u}{\partial \nu}-\beta u=0 & \text { on } \partial \Omega\end{cases}
$$

(here $\nu$ is the outer normal on $\partial \Omega$ ), i.e., in the weak sense:

$$
\int_{\Omega} D u \cdot D v \mathrm{~d} x-\beta \int_{\partial \Omega} u v \mathrm{~d} \mathcal{H}^{n-1}=\lambda \int_{\Omega} u v \mathrm{~d} x \quad \forall v \in H^{1}(\Omega)
$$

Keywords and phrases: Robin eigenvalue, quantitative isoperimetric inequality, convex sets.

1 Universitá del Salento, Dipartimento di Matematica e Fisica "Ennio De Giorgi", Via per Arnesano, 73100 Lecce, Italy.

${ }^{2}$ University of Jyväskylä, Department of Mathematics and Statistics, P.O. Box 35 (MaD), 40014, Jyväskylä, Finland.

* Corresponding author: simone.cito@unisalento.it; domenico.a.lamanna@jyu.fi 
In order to handle the first Robin eigenvalue with negative boundary parameter $-\beta$ in $\Omega$ (for brevity denoted by $\lambda_{\beta}(\Omega)$ ), it is very useful to use the following variational representation

$$
\lambda_{\beta}(\Omega)=\min _{u \in H^{1}(\Omega) \backslash\{0\}} \frac{\int_{\Omega}|D u|^{2} \mathrm{~d} x-\beta \int_{\partial \Omega} u^{2} \mathrm{~d} \mathcal{H}^{n-1}}{\int_{\Omega} u^{2} \mathrm{~d} x} .
$$

Notice that, for any Lipschitz domain $\Omega, \lambda_{\beta}(\Omega)$ is negative. Indeed, using the characteristic function of $\Omega$ as a test function in (1.2), one obtains

$$
\lambda_{\beta}(\Omega) \leq-\beta \frac{\mathcal{H}^{n-1}(\partial \Omega)}{|\Omega|}<0
$$

In particular, the map $\Omega \mapsto \lambda_{\beta}(\Omega)$ is bounded from above and unbounded from below (it is sufficient to consider a sequence of Lipschitz domains $\left(\Omega_{j}\right)_{j}$ having prescribed measure and rapidly oscillating boundaries such that $\left.\mathcal{H}^{n-1}(\partial \Omega) \rightarrow+\infty\right)$. Then, in terms of shape optimization problems, it makes sense to look for maximizers of $\lambda_{\beta}$ in suitable classes of sets.

In this paper we deal with the maximization of $\lambda_{\beta}$ among convex sets with prescribed perimeter, i.e. with problem

$$
\sup \left\{\lambda_{\beta}(\Omega): \Omega \subset \mathbb{R}^{n}, \text { open, convex, } \mathcal{H}^{n-1}(\partial \Omega)=m\right\}
$$

(the choice of the surface area constraint is more natural in our framework than the classical upper bound on the Lebesgue measure of the admissible sets, as it will be clarified in the following).

In [10] authors prove that the ball is the only maximizer of problem (1.3); more precisely, they proved the reverse Faber-Krahn inequality

$$
\lambda_{\beta}\left(\Omega^{\#}\right) \geq \lambda_{\beta}(\Omega)
$$

where $\Omega \subset \mathbb{R}^{n}$ is an open bounded convex set, $\Omega^{\#} \subset \mathbb{R}^{n}$ is the ball having the same surface area of $\Omega$ and the equality holds if and only if $\Omega$ itself is a ball.

A natural question is whether inequality (1.4) can be proved in a quantitative form, in other words if it holds some inequality like

$$
\lambda_{\beta}\left(\Omega^{\#}\right)-\lambda_{\beta}(\Omega) \geq \mathcal{A}(\Omega)^{\alpha},
$$

where $\alpha>0$ the map $\Omega \rightarrow \mathcal{A}(\Omega)$ "quantifies" in some sense how much $\Omega$ is "far" from the ball. In this paper we answer positively to the question, and the main result is the following stability issue.

Theorem 1.1. Let $n \geq 2, \rho>0$ and $\beta>0$. Then, there exist two positive constants $C(n, \rho, \beta)>0$ and $\delta_{0}(n, \rho, \beta)>0$, depending only on the dimension $n$, on the boundary parameter $\beta$ and on $\rho$, such that, for all $\Omega \subset \mathbb{R}^{n}$ with $P(\Omega)=n \omega_{n} \rho^{n-1}$ and $\lambda_{\beta}\left(\Omega^{\#}\right)-\lambda_{\beta}(\Omega) \leq \delta_{0}$, it holds

$$
\lambda_{\beta}\left(\Omega^{\#}\right)-\lambda_{\beta}(\Omega) \geq C(n, \rho, \beta) g\left(\mathcal{A}_{\mathcal{H}}(\Omega)\right)
$$


where $\Omega^{\#}$ is the only ball with $P(\Omega)=P\left(\Omega^{\#}\right)$ and whose center coincides with the barycenter of $\Omega, \mathcal{A}_{\mathcal{H}}$ is the Hausdorff asymmetry defined in $(2.2)$ and $g$ is defined by

$$
g(s)= \begin{cases}s^{2} & \text { if } n=2 \\ f^{-1}\left(s^{2}\right) & \text { if } n=3 \\ s^{\frac{n+1}{2}} & \text { if } n \geq 4\end{cases}
$$

where $f(t)=\sqrt{t \log \left(\frac{1}{t}\right)}$ for $0<t<e^{-1}$.

The interest of our stability result is also linked to the uncontrolled behaviour of the map $\Omega \rightarrow \lambda_{\beta}(\Omega)$ whenever the variable $\Omega$ rescales (see, for instance, [11]) and to the lack of general monotonicity properties. Indeed, differently from the Steklov case (see [18]) or the Dirichlet case (see, for instance, [17]), we cannot reduce to some equivalent scale invariant problem.

In [9] it has been proved that, for any $\beta>0$, a maximizing set for the $k$-th Robin eigenvalue exists in the class of sets of finite perimeter with prescribed measure; moreover, optimal sets have perimeter and diameters uniformly controlled by the parameters of the problem (the measure of the admissible sets, the dimension of the space, the order of the eigenvalue and the boundary parameter). Using a similar technique, a general existence result has been proved in [10] replacing the prescription on the measure by a constraint on the perimeter (that, in general, is not saturated).

Notice that also the constant in (1.5) depends on the uniform parameters of the problem (the prescribed perimeter, the dimension of the space and the boundary parameter); we expected this dependence in view of the above mentioned results of existence of optimal shapes and geometric control of the Robin spectrum.

Moreover, up to our knowledge, our stability result is the first proved for negative eigenvalues of the Laplace operator.

Following the approach of [14], where a reverse Faber-Krahn inequality is proven for sets of prescribed measure, our strategy is based on the study of the stability of an auxiliary Steklov-type shape optimization problem.

The paper is structured as follows. In Section 2 we present some mathematical tools needed in the following and recall some impotant results in the framework of the shape optimization of the Robin eigenvalues with negative boundary parameter; in particular, we explain why the "right" constraint to consider is on the perimeter and not on the volume of the admissible sets. In Section 3 we prove Theorem 1.1 after introducing a Steklov-type auxiliary problem and proving a stability result for it. Finally, in Section 4, we remark a sharpness issue of a related stability result and show some open problems arising from our analysis.

\section{Notations AND PRELIMINARY RESUlts}

Throughout the paper, the unit ball centered at the origin will be denoted by $B$ and its boundary by $\mathbb{S}^{n-1}$; moreover, we will denote by $B_{R}$ the ball centered at the origin of radius $R$ and by $B_{R}(x)$ the ball centered at $x$ of radius $R$.

Let $\Omega \subseteq \mathbb{R}^{n}$ be a bounded, open set and let $E \subseteq \mathbb{R}^{n}$ be a measurable set. For the sake of completeness, we recall here the definition of the perimeter of $E$ in $\Omega$ :

$$
P(E ; \Omega)=\sup \left\{\int_{E} \operatorname{div} \varphi \mathrm{d} x: \varphi \in C_{c}^{\infty}\left(\Omega ; \mathbb{R}^{n}\right),\|\varphi\|_{\infty} \leq 1\right\}
$$

The perimeter of $E$ in $\mathbb{R}^{n}$ will be denoted by $P(E)$ and, if $P(E)<\infty$, we say that $E$ is a set of finite perimeter. Moreover, if $E$ has Lipschitz boundary, we have that

$$
P(E)=\mathcal{H}^{n-1}(\partial E)
$$


where $\mathcal{H}^{n-1}$ is the $(n-1)$-dimensional Hausdorff measure in $\mathbb{R}^{n}$.

We briefly recall the notion of Hausdorff distance between compact or open bounded sets (see for instance [19]).

Definition 2.1 (Hausdorff distance and convergence). The Hausdorff distance between two non-empty compact sets $E, F \subset \mathbb{R}^{n}$ is defined by:

$$
d^{\mathcal{H}}(E, F)=\inf \left\{\varepsilon>0: E \subset F+B_{\varepsilon}, F \subset E+B_{\varepsilon}\right\} .
$$

If $D \subset \mathbb{R}^{n}$ is a compact set and $E, F \subset D$ are two bounded open sets, we define the Hausdorff distance between the two open sets $E$ and $F$ by

$$
d_{\mathcal{H}}(E, F):=d^{\mathcal{H}}(D \backslash E, D \backslash F) .
$$

This last definition is independent of the "big compact box" $D$. We say that a sequence of compact (respectively bounded open) sets $\left(E_{j}\right)_{j}$ converges to the compact (respectively bounded open) set $E$ in the sense of Hausdorff if $d^{\mathcal{H}}\left(E_{j}, E\right) \rightarrow 0$ (respectively $d_{\mathcal{H}}\left(E_{j}, E\right) \rightarrow 0$ ).

Notice that, if $E$ and $F$ are open convex sets, we have

$$
d_{\mathcal{H}}(E, F)=d^{\mathcal{H}}(\bar{E}, \bar{F})=d^{\mathcal{H}}(\partial E, \partial F)
$$

and the following rescaling property holds

$$
d_{\mathcal{H}}(t E, t F)=t d_{\mathcal{H}}(E, F), \quad t>0 .
$$

Let $\Omega \subset \mathbb{R}^{n}$ be a bounded, open, convex set. We consider the following Hausdorff asymmetry functional related to $\Omega$ :

$$
\mathcal{A}_{\mathcal{H}}(\Omega)=\min _{x \in \mathbb{R}^{n}}\left\{d_{\mathcal{H}}\left(\Omega, B_{R}(x)\right), P(\Omega)=P\left(B_{R}(x)\right)\right\}
$$

Now, let us recall another useful notion of convergence of sets.

Definition 2.2 (convergence in measure). Let $\Omega \subseteq \mathbb{R}^{n}$ be a bounded, open set, let $\left(E_{j}\right)_{j}$ be a sequence of measurable sets in $\mathbb{R}^{n}$ and let $E \subset \mathbb{R}^{n}$ be a measurable set. We say that $\left(E_{j}\right)_{j}$ converges in measure in $\Omega$ to $E$, and we write $E_{j} \rightarrow E$, if $\chi_{E_{j}} \rightarrow \chi_{E}$ in $L^{1}(\Omega)$, or in other words, if $\lim _{j \rightarrow \infty} V\left(\left(E_{j} \Delta E\right) \cap \Omega\right)=0$.

We recall also that the perimeter is lower semicontinous with respect to the local convergence in measure (see [2], Prop. 3.38), i.e. if the sequence of sets $\left(E_{j}\right)_{j}$ converges in measure in $\Omega$ to $E$, then

$$
P(E ; \Omega) \leq \liminf _{j \rightarrow \infty} P\left(E_{j} ; \Omega\right) .
$$

As a consequence of the Rellich-Kondrachov theorem, the following compactness result holds; for a reference see for instance ([2], Thm. 3.39).

Proposition 2.3. Let $\Omega \subseteq \mathbb{R}^{n}$ be a bounded, open set and let $\left(E_{j}\right)$ be a sequence of measurable sets of $\mathbb{R}^{n}$, such that $\sup _{j} P\left(E_{j} ; \Omega\right)<\infty$. Then, there exists a subsequence $\left(E_{j_{k}}\right)$ converging in measure in $\Omega$ to a set $E$, such that

$$
P(E ; \Omega) \leq \liminf _{k \rightarrow \infty} P\left(E_{j_{k}} ; \Omega\right)
$$


Another useful property concerning the sets of finite perimeter is stated in the next approximation result, see ([2], Thm. 3.42).

Proposition 2.4. Let $\Omega \subseteq \mathbb{R}^{n}$ be a bounded, open set and let $E$ be a set of finite perimeter in $\Omega$. Then, there exists a sequence of smooth, bounded open sets $\left(E_{j}\right)$ converging in measure in $\Omega$ and such that $\lim _{j \rightarrow \infty} P\left(E_{j} ; \Omega\right)=$ $P(E ; \Omega)$.

In the particular case of convex sets, the following lemma holds.

Lemma 2.5. Let $\left(E_{j}\right)_{j}$ be a sequence of convex sets in $\mathbb{R}^{n}$ such that $E_{j} \rightarrow B$ in measure, then $\lim _{j \rightarrow \infty} P\left(E_{j}\right)=$ $P(B)$.

Proof. Since, in the case of convex sets, the convergence in measure implies the Hausdorff convergence, we have that $\lim _{j \rightarrow \infty} d_{\mathcal{H}}\left(E_{j}, B\right)=0$ (see for instance [13]). Thus, for $j$ large enough, there exists $\varepsilon_{j}$, such that

$$
\left(1-\varepsilon_{j}\right) B \subset E_{j} \subset\left(1+\varepsilon_{j}\right) B
$$

Being the perimeter monotone with respect to the inclusion of convex sets then

$$
\left(1-\varepsilon_{j}\right)^{n-1} P(B) \leq P\left(E_{j}\right) \leq\left(1+\varepsilon_{j}\right)^{n-1} P(B) .
$$

When $j$ goes to infinity, we have the thesis.

Now we recall two useful results, whose proof can be found in $[16,17]$.

Lemma 2.6. If $v \in W^{1, \infty}\left(\mathbb{S}^{n-1}\right)$ and $\int_{\mathbb{S}^{n-1}} v \mathrm{~d} \mathcal{H}^{n-1}=0$, then

$$
\|v\|_{L^{\infty}\left(\mathbb{S}^{n-1}\right)}^{n-1} \leq \begin{cases}\pi\left\|D_{\tau} v\right\|_{L^{2}\left(\mathbb{S}^{n-1}\right)} & n=2 \\ 4\left\|D_{\tau} v\right\|_{L^{2}\left(\mathbb{S}^{n-1}\right)}^{2} \log \frac{8 e\left\|D_{\tau} v\right\|_{L^{\infty}\left(\mathbb{S}^{n-1}\right)}^{n-1}}{\left\|D_{\tau} v\right\|_{L^{2}\left(\mathbb{S}^{n-1}\right)}^{2}} & n=3 \\ C(n)\left\|D_{\tau} v\right\|_{L^{2}\left(\mathbb{S}^{n-1}\right)}^{2}\left\|D_{\tau} v\right\|_{L^{\infty}\left(\mathbb{S}^{n-1}\right)}^{n-3} & n \geq 4\end{cases}
$$

For this second lemma see for instance [17].

Lemma 2.7. Let $n \geq 2$. There exists a positive universal constant $\varepsilon_{0}<\frac{1}{2}$ such that, if $E$ is a convex, nearly spherical set with $V(E)=V(B)$ and $\|v\|_{W^{1, \infty}} \leq \varepsilon_{0}$, then

$$
\left\|D_{\tau} v\right\|_{L^{\infty}}^{2} \leq 8\|v\|_{L^{\infty}}
$$

\subsection{Nearly spherical sets}

In this section we give the definition of nearly spherical sets and we recall some of their basic properties (see for instance $[6,16,17])$.

Definition 2.8. Let $n \geq 2$. An open, bounded set $E \subset \mathbb{R}^{n}$ is said a nearly spherical set parametrized by $v$ if the barycenter of $E$ is at the origin and there exists $v \in W^{1, \infty}\left(\mathbb{S}^{n-1}\right)$ such that

$$
\partial E=\left\{y \in \mathbb{R}^{n}: y=x(1+v(x)), x \in \mathbb{S}^{n-1}\right\},
$$

with $\|v\|_{W^{1, \infty}} \leq \frac{1}{2}$. 


\subsection{The Robin eigenvalues with negative boundary parameter}

In this short paragraph we briefly recall some well known properties and shape optimization results for Robin eigenvalues with negative boundary parameter. For more details see, for instance, [11].

As already highlighted, for any Lipschitz domain $\Omega, \lambda_{\beta}(\Omega)$ is negative; moreover, if $\Omega$ is connected, $\lambda_{\beta}(\Omega)$ is simple. In addiction, for any scale factor $t \geq 0$, one has

$$
\lambda_{\beta}(t \Omega)=\frac{1}{t^{2}} \lambda_{t \beta}(\Omega)
$$

notice that this scaling formula gives no scale invariance of the functional $\lambda_{\beta}$ and no monotonicity properties, since also the boundary parameter rescales. This uncontrolled behaviour when the set rescales does not allow to reduce to equivalent scale invariant problems (for instance, to reduce ourselves to study problem (1.3) only in the class of convex sets with unitary perimeter).

For many years it has been studied the maximization of $\lambda_{\beta}$ among sets of prescribed measure, inspired by a conjecture by M. Bareket of 1977 (see [4] for the original statement in dimension $n=2$ and [7] for a more general statement in any dimension): it was conjectured that the ball was the only maximizer for $\lambda_{\beta}$ among suficiently smooth sets of prescribed measure for any value of $\beta$ and any dimension $n$. Even though there was some evidence supporting the conjecture, in 2015 the conjecture was disproved in [15]. More precisely, authors prove that for any $r>0$, there exist a spherical shell $A_{r_{1}, r_{2}}:=\left\{x \in \mathbb{R}^{d}: r_{1}<|x|<r_{2}\right\}$ with the same volume as the ball $B_{r}$, such that

$$
\lambda_{\beta}\left(B_{r}\right)<\lambda_{\beta}\left(A_{r_{1}, r_{2}}\right)
$$

for every sufficiently large value of $\beta$. On the other hand, for smooth domains in the plane, a reverse FaberKrahn inequality holds for small negative values of $\beta$ (see Thm. 2 in [15]): for every $m>0$, there exist $\beta_{0}>0$ depending only on $m$ such that, for any bounded domain $\Omega$ of measure $m$ with $C^{2}$ boundary

$$
\lambda_{\beta}(\Omega) \leq \lambda_{\beta}\left(B_{m}\right)
$$

for every $\beta \in\left[0, \beta_{0}\right]$, where $B_{m}$ is the disk of measure $m$.

In terms of existence of a maximizer for $\lambda_{\beta}$ in any space dimension and for any value of $\beta$, in [9] it is proved that among sets of prescribed measure a solution exists (also for higher eigenvalues and for more general functional), but nothing is known about the precise shape of the optimal sets.

Since for a quantitative approach it is necessary to know what is the (unique) optimal set, it has been natural to replace the prescription of fixed measure with the prescription of fixed perimeter and to restrict the class of admissible sets. More precisely, in [10] authors prove the reverse Faber-Krahn inequality (1.4), i.e. that the ball is the only maximizer for $\lambda_{\beta}$ among convex sets of fixed perimeter. In view of that uniqueness, the prescription on the perimeter and the convexity constraint on the admissible sets turn out to be two reasonable requirements to study the stability of inequality (1.4). In the next section we focus on this quantitative result, the main goal of our work.

\section{A stability Result for a Steklov-type Problem and Proof OF THEOREM 1.1}

This section is the core of the paper. We first introduce the auxiliary problem (3.3) and then present a stability inequality for it. Our approach follows the ideas in [18]. Finally, we prove Theorem 1.1 as a consequence of the quantitative reverse Faber-Krahn type inequality for (3.3). 
Let $n \geq 2, \Omega \subset \mathbb{R}^{n}$ and consider the following variational problem

$$
\lambda(\Omega)=\inf _{u \in H^{1} \backslash\{0\}} \frac{\int_{\Omega}|D u|^{2} \mathrm{~d} x+\int_{\Omega} u^{2} \mathrm{~d} x}{\int_{\partial \Omega} u^{2} \mathrm{~d} \mathcal{H}^{n+1}} .
$$

It is well known that if $\Omega$ is an open, bounded set with Lipschitz boundary the infimum in (3.1) is achieved and it solves the following Steklov-type eigenvalue problem

$$
\begin{cases}-\Delta v+v=0 & \text { in } \Omega \\ \frac{\partial v}{\partial \nu}=\lambda(\Omega) v & \text { on } \partial \Omega\end{cases}
$$

In [14] it is highlighted that the shape optimization problem

$$
\sup \left\{\lambda(\Omega): \Omega \subset \mathbb{R}^{n} \text {, open, convex, } \mathcal{H}^{n-1}(\partial \Omega)=m\right\}
$$

is linked with the maximization of $\lambda_{\beta}$ in a particular class of open sets with fixed measure; the proof that the ball maximizes $\lambda_{\beta}$ is obtained via means of quantitative isoperimetric inequalities.

Here we state some well known facts for $\lambda(\Omega)$. If $\Omega$ is the unit ball, the only solution of the problem (3.1), up to a multiplicative constant, is given by $z(x)=|x|^{1-\frac{n}{2}} \mathcal{I}_{\frac{n}{2}-1}(|x|)$, where with $\mathcal{I}_{\nu}$ we indicate the modified Bessel function of index $\nu$ for every $\nu \in \mathbb{R}$.

Following the notations in [14], we now introduce the functions

$$
h_{\rho}(t)=\left((t \rho)^{1-\frac{n}{2}} \mathcal{I}_{\frac{n}{2}-1}(t \rho)\right)^{2} ; \quad f_{\rho}(t)=\frac{h_{\rho}^{\prime}(t)}{2 \rho}=(t \rho)^{2-n} \mathcal{I}_{\frac{n}{2}-1}(t \rho) \mathcal{I}_{\frac{n}{2}}(t \rho) .
$$

Since the minimizer of (3.1) is known to be $z(x)$ in case $\Omega$ is any ball centered at the origin, the idea is to test $\lambda(\Omega)$ against $z(x)$. This leads to prove that the only solution to problem (3.3) is the ball, as stated in the following

Lemma 3.1. Let $n \geq 2$ and $\Omega \subset \mathbb{R}^{n}$ be open and convex. Then

$$
\lambda(\Omega) \leq \frac{\int_{\partial \Omega} z \frac{\partial z}{\partial \nu} \mathrm{d} \mathcal{H}^{n-1}}{\int_{\partial \Omega} z^{2}}=: \frac{N(\Omega)}{D(\Omega)}=: I(\Omega) \quad \text { and } \quad I\left(\Omega^{\#}\right)=\lambda\left(\Omega^{\#}\right)
$$

where $z(x)=|x|^{1-\frac{n}{2}} \mathcal{I}_{\frac{n}{2}-1}(|x|)$.

The proof of this lemma is an easy consequence of the divergence theorem, the variational formulation of $\lambda$ and the fact that $-\Delta z+z=0$.

In order to write the ratio $N(\Omega) / V(\Omega)$ for nearly spherical sets, we recall that the tangential Jacobian of the $\operatorname{map} \phi: x \in \mathbb{S}^{n-1} \rightarrow y=\rho x(1+u(x))$ is given by

$$
J_{\phi}(x)=\rho^{n-1}(1+u)^{n-2} \sqrt{(1+u)^{2}+\left|D_{\tau} u\right|^{2}}
$$


where with $D_{\tau} u$ we indicate the tangential gradient of $u$, while the exterior normal to $\partial \Omega$ at $y=x(1+u(x))$ can be written as

$$
\nu(x)=\frac{x(1+u(x))-D_{\tau} u(x)}{\sqrt{(1+u(x))^{2}+\left|D_{\tau} u(x)\right|^{2}}} .
$$

Hence recalling the explicit formula for $z$, i.e. $z(x)=|x|^{1-\frac{n}{2}} \mathcal{I}_{\frac{n}{2}-1}(|x|)$, and using the area formula we can write the ratio $N(\Omega) / D(\Omega)$ for nearly spherical sets as

$$
\frac{N(\Omega)}{D(\Omega)}=\frac{\int_{\mathbb{S}^{n-1}} f_{\rho}(1+u(x))(1+u(x))^{n-1} \mathrm{~d} \mathcal{H}^{n-1}}{\int_{\mathbb{S}^{n-1}} h_{\rho}(1+u(x))(1+u(x))^{n-1} \sqrt{(1+u(x))^{2}+\left|D_{\tau} u(x)\right|^{2}} \mathrm{~d} \mathcal{H}^{n-1}} .
$$

Proposition 3.2. Let $n \geq 2$ and $\rho>0$. There exist $\varepsilon_{0}>0$ and $a C_{1}(n, \rho)>0$ such that, for any nearly spherical set as in the definition 2.5 with $\|v\|_{\infty} \leq \varepsilon_{0}$, the following stability inequality holds true

$$
\frac{N\left(\Omega^{\#}\right) D(\Omega)-D\left(\Omega^{\#}\right) N(\Omega)}{n \omega_{n}} \geq C_{1}(n, \rho)\left\|D_{\tau} v\right\|_{L^{2}\left(\mathbb{S}^{n-1}\right)}^{2}
$$

Proof. Let the idea is to estimate from below the left hand side of (3.6) by performing a suitable "Taylor expansion" in terms of the deformation of $\partial \Omega$ compared to $\mathbb{S}^{n-1}$. For that reason, it is convenient to replace the polar representation $v$ of $\partial \Omega$ by $t u$ where $t>0$ is sufficiently small. We thus obtain

$$
\begin{aligned}
& \frac{N\left(\Omega^{\#}\right) D(\Omega)-D\left(\Omega^{\#}\right) N(\Omega)}{n \omega_{n}} \\
& =f_{\rho}(1) \int_{\mathbb{S}^{n-1}} h_{\rho}(1+t u)(1+t u)^{n-1} \sqrt{1+\frac{t^{2}\left|D_{\tau} u\right|^{2}}{(1+t u)^{2}}} \mathrm{~d} \sigma-h_{\rho}(1) \int_{\mathbb{S}^{n-1}} f_{\rho}(1+t u)(1+t u)^{n-1} \mathrm{~d} \sigma .
\end{aligned}
$$

By a Taylor expansion up to the second order around $t=0$ of the two integrals, we get

$$
\begin{aligned}
& \frac{N\left(\Omega^{\#}\right) D(\Omega)-D\left(\Omega^{\#}\right) N(\Omega)}{n \omega_{n}} \\
& \geq \int_{\mathbb{S}^{n-1}} t u\left[f_{\rho}(1) h_{\rho}^{\prime}(1)-f_{\rho}^{\prime}(1) h_{\rho}(1)\right] \mathrm{d} \sigma \\
& +\int_{\mathbb{S}^{n-1}} \frac{t^{2} u^{2}}{2}\left[2(n-1)\left(f_{\rho}(1) h_{\rho}^{\prime}(1)-f_{\rho}^{\prime}(1) h_{\rho}(1)\right)+f_{\rho}(1) h_{\rho}^{\prime \prime}(1)-f_{\rho}^{\prime \prime}(1) h_{\rho}(1)\right] \mathrm{d} \sigma \\
& +\int_{\mathbb{S}^{n-1}} f_{\rho}(1) h_{\rho}(1) \frac{t^{2}\left|D_{\tau} u\right|^{2}}{2} \mathrm{~d} \sigma-\varepsilon C_{1} t^{2}\left\|D_{\tau} u\right\|_{L^{2}\left(\mathbb{S}^{n-1}\right)}^{2} \\
& \geq t^{2}\left[\frac{n}{2}\left(f_{\rho}(1) h_{\rho}^{\prime}(1)-f_{\rho}^{\prime}(1) h_{\rho}(1)\right)+\frac{f_{\rho}(1) h_{\rho}^{\prime \prime}(1)-f_{\rho}^{\prime \prime}(1) h_{\rho}(1)}{2}\right]\|u\|_{L^{2}\left(\mathbb{S}^{n-1}\right)}^{2} \\
& +t^{2}\left[\frac{f_{\rho}(1) h_{\rho}(1)}{2}-\frac{f_{\rho}(1) h_{\rho}^{\prime}(1)-f_{\rho}^{\prime}(1) h_{\rho}(1)}{2(n-1)}\right]\left\|D_{\tau} u\right\|_{L^{2}\left(\mathbb{S}^{n-1}\right)}^{2}-\varepsilon C_{2} t^{2}\left\|D_{\tau} u\right\|_{L^{2}\left(\mathbb{S}^{n-1}\right)}^{2}
\end{aligned}
$$

Now we make use of the hypothesis that $P(\Omega)=P\left(\Omega^{\#}\right)$. In term of integrals, this means

$$
\int_{\mathbb{S}^{n-1}}(1+u)^{n-2} \sqrt{(1+u)^{2}+\left|D_{\tau} u\right|^{2}} \mathrm{~d} \mathcal{H}^{n-1}=n \omega_{n}
$$


Using the fact that $t<\varepsilon$, we can Taylor expand the LHS and find

$$
t \int_{\mathbb{S}^{n-1}} u \mathrm{~d} \mathcal{H}^{n-1} \geq-t^{2} \frac{n-2}{2} \int_{\mathbb{S}^{n-1}} u^{2} \mathrm{~d} \mathcal{H}^{n-1}-t^{2} \frac{1}{2(n-1)} \int_{\mathbb{S}^{n-1}}\left|D_{\tau} u\right|^{2} \mathrm{~d} \mathcal{H}^{n-1}-C_{3}(n) o\left(t^{2}\right)
$$

Notice that

$$
\left[\frac{f_{\rho}(1) h_{\rho}(1)}{2}-\frac{f_{\rho}(1) h_{\rho}^{\prime}(1)-f_{\rho}^{\prime}(1) h_{\rho}(1)}{2(n-1)}\right]=\frac{\rho\left(\mathcal{I}_{\frac{n}{2}}^{2}-1(\rho)-\mathcal{I}_{\frac{n}{2}}^{2}(\rho)\right)}{2(n-1) \mathcal{I}_{\frac{n}{2}}^{2}(\rho)}+\frac{\mathcal{I}_{\frac{n}{2}-1}(\rho)}{2 \mathcal{I}_{\frac{n}{2}}(\rho)}>0
$$

Then, since $\varepsilon C_{2}$ can be taken arbitrarily small (the quantity $C_{2}$ does not depend on $\varepsilon$ ), if

$$
\left[\frac{n}{2}\left(f_{\rho}(1) h_{\rho}^{\prime}(1)-f_{\rho}^{\prime}(1) h_{\rho}(1)\right)+\frac{f_{\rho}(1) h_{\rho}^{\prime \prime}(1)-f_{\rho}^{\prime \prime}(1) h_{\rho}(1)}{2}\right] \geq 0,
$$

the proof is concluded (the positive constant in the statement is provided by (3.9)). Otherwise, we have to make a careful estimate in order to understand which term is the dominant one. To do that, let us recall that the space $L^{2}\left(\mathbb{S}^{n-1}\right)$ admits the set of spherical harmonics $\left\{Y_{k, i}, 1 \leq i \leq N_{k}, k \in \mathbb{N}\right\}$ as orthonormal basis. To be more precise, for any $k \in \mathbb{N}$, the eigenvalue problem

$$
-\Delta_{\mathbb{S}^{n-1}} Y_{k, i}=n(n-k-2) Y_{k, i},
$$

where $\Delta_{\mathbb{S}^{n-1}}$ is the Laplace Beltrami operator, admits $N_{k}$ independent solutions, called the spherical harmonics of order $k$, and we will indicate them as $\left\{Y_{k, i}, 1 \leq i \leq N_{k}\right\}$. Moreover, it is well known that the spherical harmonics of order $k$ are nothing else than homogeneous polynomials of degree $k$. Hence, we will write $u$ as

$$
u=\sum_{k=1}^{\infty} \sum_{i=1}^{N_{k}} a_{k, i} Y_{k, i}
$$

where $a_{k, i}=\int_{\mathbb{S}^{n-1}} u Y_{k, i}$. Since $\left\{Y_{k, i}, 1 \leq i \leq N_{k}, k \in \mathbb{N}\right\}$ is an orthonormal basis we have

$$
\|u\|_{L^{2}\left(\mathbb{S}^{n-1}\right)}^{2}=a_{0}^{2}+\sum_{k=0}^{\infty} \sum_{i=1}^{N_{k}} a_{k, i}^{2}
$$

and using the properties of $Y_{k, i}$ it holds

$$
\left\|D_{\tau} u\right\|_{L^{2}\left(\mathbb{S}^{n-1}\right)}^{2}=\sum_{k=1}^{\infty} \sum_{i=1}^{N_{k}} k(n+k-2) a_{k, i}^{2} .
$$

Since $Y_{0}$ is a constant and $Y_{1, i}=x_{i}$ for $1 \leq i \leq n$, we now estimate $a_{0}$ and $a_{1, i}$ using the geometric hypothesis on $\Omega$. Indeed, using (3.8) and the fact that $t \leq \varepsilon_{0}$ we infer

$$
\left|a_{0}\right|=\left|\int_{\mathbb{S}^{n-1}} u\right| \leq t \frac{n-2}{2}\|u\|_{L^{2}\left(\mathbb{S}^{n-1}\right)}^{2}+t \frac{1}{2(n-2)}\left\|D_{\tau} u\right\|_{L^{2}\left(\mathbb{S}^{n-1}\right)}^{2} .
$$


Moreover, since $B(\Omega)=0$, we have

$$
\int_{\mathbb{S}^{n-1}} x_{i}(1+t u(x))^{n+1} \mathrm{~d} \mathcal{H}^{n-1}=0
$$

and then

$$
\left|a_{1, i}\right|=\left|\int_{\mathbb{S}^{n-1}} x_{i} u(x) \mathrm{d} \mathcal{H}^{n-1}\right| \leq n \varepsilon\|u\|_{L^{2}\left(\mathbb{S}^{n-1}\right)}^{2}
$$

Thus,

$$
\|u\|_{L^{2}\left(\mathbb{S}^{n-1}\right)}^{2}=a_{0}^{2}+\sum_{i=1}^{n} a_{1, i}^{2}+\sum_{k=2}^{\infty} \sum_{i=2}^{N_{k}} a_{1, i}^{2} \sum_{k=0}^{\infty} \sum_{i=1}^{N_{k}} a_{k, i}^{2} \leq C_{n} \varepsilon^{2}\left(\|u\|_{W^{1,2}\left(\mathbb{S}^{n-1}\right)}^{2}\right)+\sum_{k=2}^{\infty} \sum_{i=2}^{N_{k}} a_{k, i}^{2}
$$

while

$$
\left\|D_{\tau} u\right\|_{L^{2}\left(\mathbb{S}^{n-1}\right)}^{2}=\sum_{k=1}^{\infty} \sum_{i=1}^{i=N_{k}} a^{2} \geq 2 n \sum_{k=2}^{\infty} \sum_{i=2}^{N_{k}} a_{k, i}^{2}
$$

If we combine the above two inequalities we get that

$$
\left\|D_{\tau} u\right\|_{L^{2}\left(\mathbb{S}^{n-1}\right)}^{2} \geq \frac{2 n-\varepsilon}{1-\varepsilon}\|u\|_{L^{2}\left(\mathbb{S}^{n-1}\right)}^{2}=\left(2 n-\frac{2 \varepsilon}{1-\varepsilon}\right)\|u\|_{L^{2}\left(\mathbb{S}^{n-1}\right)}^{2} .
$$

Using (3.7) and (3.10) we have

$$
\begin{aligned}
& \frac{N\left(\Omega^{\#}\right) D(\Omega)-D\left(\Omega^{\#}\right) N(\Omega)}{n \omega_{n}} \\
& \geq \frac{t^{2}}{2 n}\left[\frac{n}{2}\left(f_{\rho}(1) h_{\rho}^{\prime}(1)-f_{\rho}^{\prime}(1) h_{\rho}(1)\right)+\frac{f_{\rho}(1) h_{\rho}^{\prime \prime}(1)-f_{\rho}^{\prime \prime}(1) h_{\rho}(1)}{2}\right]\left\|D_{\tau} u\right\|_{L^{2}\left(\mathbb{S}^{n-1}\right)}^{2} \\
& +t^{2}\left[\frac{f_{\rho}(1) h_{\rho}(1)}{2}-\frac{f_{\rho}(1) h_{\rho}^{\prime}(1)-f_{\rho}^{\prime}(1) h_{\rho}(1)}{2(n-1)}\right]\left\|D_{\tau} u\right\|_{L^{2}\left(\mathbb{S}^{n-1}\right)}^{2}-\varepsilon C_{2} t^{2}\left\|D_{\tau} u\right\|_{L^{2}\left(\mathbb{S}^{n-1}\right)}^{2} \\
& \geq \frac{t^{2}}{2 n}\left[\frac{n^{2}-3 n}{2(n-1)}\left(f_{\rho}(1) h_{\rho}^{\prime}(1)-f_{\rho}^{\prime}(1) h_{\rho}(1)\right)+\frac{f_{\rho}(1) h_{\rho}^{\prime \prime}(1)-f_{\rho}^{\prime \prime}(1) h_{\rho}(1)}{2}\right. \\
& \left.+n\left(f_{\rho}(1) h_{\rho}(1)\right)\right]\|u\|_{L^{2}\left(\mathbb{S}^{n-1}\right)}^{2}-\varepsilon C_{2} t^{2}\left\|D_{\tau} u\right\|_{L^{2}\left(\mathbb{S}^{n-1}\right)}^{2} .
\end{aligned}
$$

To conclude the proof we are left to show that

$$
\frac{n^{2}-3 n}{n-1}\left(f_{\rho}(1) h_{\rho}^{\prime}(1)-f_{\rho}^{\prime}(1) h_{\rho}(1)\right)+f_{\rho}(1) h_{\rho}^{\prime \prime}(1)-f_{\rho}^{\prime \prime}(1) h_{\rho}(1)+2 n\left(f_{\rho}(1) h_{\rho}(1)\right)>0 .
$$

Now, in [14] authors prove that

$$
C(n, \rho)=(n-1)\left(f_{\rho}(1) h_{\rho}^{\prime}(1)-f_{\rho}^{\prime}(1) h_{\rho}(1)\right)+f_{\rho}(1) h_{\rho}^{\prime \prime}(1)-f_{\rho}^{\prime \prime}(1) h_{\rho}(1)+2 n\left(f_{\rho}(1) h_{\rho}(1)\right)>0 .
$$


for any $n \geq 2$ and $\rho>0$. Since it holds

$$
\begin{aligned}
& \frac{n^{2}-3 n}{n-1}\left(f_{\rho}(1) h_{\rho}^{\prime}(1)-f_{\rho}^{\prime}(1) h_{\rho}(1)\right)+f_{\rho}(1) h_{\rho}^{\prime \prime}(1)-f_{\rho}^{\prime \prime}(1) h_{\rho}(1)+2 n\left(f_{\rho}(1) h_{\rho}(1)\right) \\
& =C(n, \rho)-\frac{n+1}{n-1}\left(f_{\rho}(1) h_{\rho}^{\prime}(1)-f_{\rho}^{\prime}(1) h_{\rho}(1)\right)
\end{aligned}
$$

to prove (3.12) it is sufficient to show that

$$
\left(f_{\rho}(1) h_{\rho}^{\prime}(1)-f_{\rho}^{\prime}(1) h_{\rho}(1)\right) \leq 0
$$

for any $n \geq 2$ and $\rho>0$. Now, in view of the definition of $f_{\rho}$ and $h_{\rho}$, it results that

$$
f_{\rho}(1) h_{\rho}^{\prime}(1)-f_{\rho}^{\prime}(1) h_{\rho}(1)=f_{\rho}(1)=f_{\rho}^{2}(1) \frac{\mathrm{d}}{\mathrm{dt}}\left[\frac{h_{\rho}(t)}{f_{\rho}(t)}\right]_{t=1}=f_{\rho}^{2}(1) \cdot \frac{\mathrm{d}}{\mathrm{dt}}\left[\frac{\mathcal{I}_{\frac{n}{2}-1}(t \rho)}{\mathcal{I}_{\frac{n}{2}}(t \rho)}\right]_{t=1} .
$$

In [3] it has been proved that the last derivative is negative; then (3.12) holds and the proof is concluded.

As an immediate application of Lemma 3.1 and Proposition 3.2, we get the following

Corollary 3.3. Let $n \geq 2$ and $\Omega$ a nearly spherical set parametrized by $u \in W^{1, \infty}\left(\mathbb{S}^{n-1}\right)$ with perimeter $P(\Omega)=$ $n \omega_{n} \rho^{n-1}$. Then

$$
\lambda\left(\Omega^{\#}\right)-\lambda(\Omega) \geq I\left(\Omega^{\#}\right)-I(\Omega) \geq C_{1}(n, \rho)\left\|D_{\tau} u\right\|_{L^{2}\left(\mathbb{S}^{n-1}\right)}^{2}
$$

Moreover, the constant $C_{1}(n, \rho)$ depends continuously (actually analytically) on $\rho$.

A second corollary, actually the one we are interested in, regards the stability of the first eigenvalue for the Robin problem.

Corollary 3.4. Let $n \geq 2$ and $\delta>0$. There exists a constant $C(n, \beta, \rho, \delta)$ such that if $\Omega$ is a nearly spherical set parametrized by $u \in W^{1, \infty}\left(\mathbb{S}^{n-1}\right)$ with $\lambda_{\beta}\left(\Omega^{\#}\right)-\lambda_{\beta}(\Omega)<\delta$, then

$$
\lambda_{\beta}\left(\Omega^{\#}\right)-\lambda_{\beta}(\Omega) \geq C(n, \beta, \rho, \delta)\left\|D_{\tau} u\right\|_{L^{2}\left(\mathbb{S}^{n-1}\right)}^{2} .
$$

Proof. Let $\delta>0$ be fixed and let $\Omega$ be chosen as in the statement. The map $\beta \mapsto \lambda_{\beta}(\Omega)$ is continuous and monotonically decreasing from $(0,+\infty)$ onto $(-\infty, 0)$. Then, there exists $\bar{\beta} \geq \beta$ such that

$$
\lambda_{\beta}(\Omega)=\lambda_{\bar{\beta}}\left(\Omega^{\#}\right) .
$$

Hence,

$$
\lambda_{\beta}\left(\Omega^{\#}\right)-\lambda_{\beta}(\Omega)=\lambda_{\beta}\left(\Omega^{\#}\right)-\lambda_{\bar{\beta}}\left(\Omega^{\#}\right)
$$

Moreover, if $\kappa=\sqrt{\left|\lambda_{\beta}(\Omega)\right|}$, for the rescaled sets $\kappa \Omega$ and $\kappa \Omega^{\#}$ it holds that

$$
\int_{\kappa \Omega}|\nabla w|^{2} \mathrm{~d} x+\int_{\kappa \Omega} w^{2} \mathrm{~d} x \geq \frac{\beta}{\kappa} \int_{\partial(\kappa \Omega)} w^{2} \mathrm{~d} \mathcal{H}^{n-1} \quad \forall w \in H^{1}(\kappa \Omega)
$$


and

$$
\int_{\kappa \Omega^{\#}}|\nabla z|^{2} \mathrm{~d} x+\int_{\kappa \Omega^{\#}} z^{2} \mathrm{~d} x \geq \frac{\bar{\beta}}{\kappa} \int_{\partial\left(\kappa \Omega^{\#}\right)} z^{2} \mathrm{~d} \mathcal{H}^{n-1} \quad \forall z \in H^{1}\left(\kappa \Omega^{\#}\right),
$$

with equality holding if $w$ and $z$ are, respectively, the eigenfunctions for the Robin problem on $\kappa \Omega$ with parameter $\beta$ and on $\kappa \Omega^{\#}$ with boundary parameter $\bar{\beta}$. In that case, the infimum for the Steklov problem (3.1) is achieved on $\kappa \Omega$ and $\kappa \Omega^{\#}$, obtaining

$$
\lambda(\kappa \Omega)=\frac{\beta}{\kappa}, \quad \lambda\left(\kappa \Omega^{\#}\right)=\frac{\bar{\beta}}{\kappa} .
$$

Then, it follows that

$$
\frac{\bar{\beta}-\beta}{\sqrt{\left|\lambda_{\beta}(\Omega)\right|}}=\lambda\left(\kappa \Omega^{\#}\right)-\lambda(\kappa \Omega)
$$

Using the variational characterization of $\lambda_{\beta}\left(\Omega^{\#}\right)$ and $\lambda_{\bar{\beta}}\left(\Omega^{\#}\right)$, if with $u_{\beta}$ we denote the optimal function of $\Omega^{\#}$ for the Robin problem with parameter $\beta$, we have that

$$
\begin{aligned}
\lambda_{\beta}\left(\Omega^{\#}\right)-\lambda_{\beta}(\Omega) & =\lambda_{\beta}\left(\Omega^{\#}\right)-\lambda_{\bar{\beta}}\left(\Omega^{\#}\right) \geq(\bar{\beta}-\beta) \frac{\int_{\partial \Omega_{\#}} u_{\beta}^{2} \mathrm{~d} \mathcal{H}^{n-1}}{\int_{\Omega^{\#}} u_{\beta}^{2} \mathrm{~d} x}=C(n, \beta, \rho)(\beta-\bar{\beta}) \\
& \geq \kappa C(n, \beta, \rho)\left(\lambda\left(\kappa \Omega^{\#}\right)-\lambda(\kappa \Omega)\right) \geq \kappa C(n, \beta, \rho) C_{1}(n, \kappa \rho)\left\|D_{\tau} u\right\|_{L^{2}\left(\mathbb{S}^{n-1}\right)}^{2},
\end{aligned}
$$

where $C_{1}$ is the constant found in Proposition 3.2.

Now we are able to prove our main stability result. Since the constant $C_{1}$ continuously depends on $\rho$ we find that

Lemma 3.5. Let $m>0$. If $\Omega$ is a convex set with with $P(\Omega)=m$ and $\lambda_{\beta}(\Omega)>2 \lambda_{\beta}\left(\Omega^{\#}\right)$, then, there exists a positive constant $C(m, n, \beta)$ such that $\operatorname{diam}(\Omega)<C(m, n, \beta)$.

Proof. Let us argue by contradiction and suppose that there exists a sequence $\left(\Omega_{j}\right)_{j}$ of convex sets with $P\left(\Omega_{j}\right)=$ $m$ such that $\lambda_{\beta}\left(\Omega_{j}\right)>2 \lambda_{\beta}\left(\Omega^{\#}\right)$ and that $\operatorname{diam}\left(\Omega_{j}\right) \rightarrow+\infty$. In view of the convexity of $\Omega_{j}$ and of the constraint $P\left(\Omega_{j}\right)=m$, the sequence $\left(\rho_{j}\right)_{j}$ of the inradii of $\left(\Omega_{j}\right)_{j}$ is necessarily vanishing. Recalling that, for any convex set $A$ with inradius $\rho$ it holds

$$
|A| \leq \rho P(A)
$$

(see, for instance, [8], Prop. 2.4.3), we deduce that $\left|\Omega_{j}\right|$ vanishes as $j$ goes to $+\infty$. Now, using $\chi_{\Omega_{j}}$ as a test function for $\lambda_{\beta}\left(\Omega_{j}\right)$, we obtain

$$
\lambda_{\beta}\left(\Omega_{j}\right) \leq-\beta \frac{P\left(\Omega_{j}\right)}{\left|\Omega_{j}\right|} \rightarrow-\infty
$$

in contradiction with the lower bound on $\lambda_{\beta}\left(\Omega_{j}\right)$.

The previous result can be even obtained as a particular case of the isodiametric control of the Robin spectrum proved in [9] also for higher eigenvalues in a wider class of sets.

Another important result about the functional $\Omega \mapsto \lambda_{\beta}(\Omega)$ is the following upper semicontinuity issue. 
Lemma 3.6. If $\Omega_{j}, \Omega \subset \mathbb{R}^{n}$ are convex sets with $\Omega_{j} \rightarrow \Omega$ is measure, then

$$
\limsup _{j \rightarrow+\infty} \lambda_{\beta}\left(\Omega_{j}\right) \leq \lambda_{\beta}(\Omega) .
$$

Sketch of the proof. As shown in [9] and in [10], the upper semicontinuity of $\lambda_{\beta}$ is based on the fact that, set $u_{\Omega}$ a (normalized) eigenfunction for $\lambda_{\beta}(\Omega)$, the functional

$$
\Omega \mapsto \int_{\partial \Omega} u_{\Omega}^{2} \mathrm{~d} \mathcal{H}^{n-1}
$$

is lower semicontinuous (for a proof of this fact in a convex setting see [12]). Thus, $\Omega \rightarrow \lambda_{\beta}(\Omega)$ is an infimum of upper semicontinous functions, which is our thesis.

In view of the upper semicontinuity of $\lambda_{\beta}$ and of the isodiametric control in Lemma 3.5, since the ball is the unique maximizer of $\lambda_{\beta}$ among convex sets with prescribed perimeter, it is immediate the following result.

Lemma 3.7. If $\Omega_{j}$ is a sequence of convex sets with barycenter at the origin and $\lambda_{\beta}\left(\Omega_{j}\right) \rightarrow \lambda_{\beta}(B)$, then $P\left(\Omega_{j}\right) \rightarrow P(B)$ and $d_{\mathcal{H}}\left(\Omega_{j}, B\right) \rightarrow 0$.

Sketch of the proof. The diameters of the sets $\Omega_{j}$ are uniformly bounded. Up to subsequences, there exists an open bounded set $\Omega$ such that $d_{\mathcal{H}}\left(\Omega_{j}, \Omega\right) \rightarrow 0$; in view of the maximality of the ball and of the upper semicontinuity of $\lambda_{\beta}$, we obtain that necessarily $\Omega=B$.

In view of the previous lemma, we can restrict our main stability result to nearly spherical sets barycentered in the origin.

Lemma 3.8. Let $n \geq 2$ and $\rho>0$. There exists a positive constant $\delta_{0}$ depending only on the dimension $n$, on the boundary parameter $\beta$ and on $\rho$ such that, if $\Omega$ is a convex set with $P(\Omega)=n \omega_{n} \rho^{n-1}$ and $\lambda_{\beta}\left(\Omega^{\#}\right)-\lambda_{\beta}(\Omega) \leq \delta_{0}$, then up to a translation $\Omega$ is a nearly spherical set.

Now we are able to prove our main result.

Proof of Theorem 1.1. Lemma 3.8 ensures us that, if $\delta_{0}$ is small enough, then we can suppose without loss of generality that our set $\Omega$ is a nearly spherical set with barycenter at the origin. Thus, we can suppose

$$
\partial \Omega=\left\{\rho x(1+u(x)), x \in \mathbb{S}^{n-1}\right\}
$$

and $P(\Omega)=n \omega_{n} \rho^{n-1}=P\left(B_{\rho}\right)$. Let $\rho_{1}$ such that $\left|B_{\rho_{1}}\right|=|\Omega|$. Since $\Omega$ and $B_{\rho_{1}}$ have the same measure, we have that

$$
\frac{\rho^{n}}{n} \int_{\mathbb{S}^{n-1}}(1+u)^{n} \mathrm{~d} \mathcal{H}^{n-1}=\omega_{n} \rho_{1}^{n}
$$

Define the function $h:=(\rho(1+u))^{n}-\rho_{1}^{n}$. Expanding the integrand of the latter formula leads to

$$
\rho^{n}-\rho_{1}^{n}=-\frac{1}{\omega_{n}} \int_{\mathbb{S}^{n-1}} \sum_{1}^{n}\left(\begin{array}{l}
n \\
k
\end{array}\right) u^{k} \mathrm{~d} \mathcal{H}^{n-1}
$$

which immediately implies $\left|\rho-\rho_{1}\right|<C(n)\|u\|_{\infty}$. Hence it holds

$$
C_{3}\|h\|_{\infty} \leq\|u\|_{\infty} \leq C_{4}\|h\|_{\infty},
$$


where $C_{3}$ and $C_{4}$ are constant depending only on the dimension. More over, the Leibnitz rule yelds $D_{\tau} h=$ $n \rho^{n}(1+u)^{n-1} D_{\tau} u$, hence to the control

$$
C_{5}\left\|D_{\tau} u\right\|_{L^{2}\left(\mathbb{S}^{n-1}\right)} \leq\left\|D_{\tau} h\right\|_{L^{2}\left(\mathbb{S}^{n-1}\right)} \leq C_{6}\left\|D_{\tau} u\right\|_{L^{2}\left(\mathbb{S}^{n-1}\right)}
$$

where $C_{5}, C_{6}$ depend on the dimension and on $\rho$. From (3.15), we know that $h$ has zero integral, thus we can apply Lemma 2.6 to $h$ and use the norm controls given above to infer

$$
\|u\|_{L^{\infty}\left(\mathbb{S}^{n-1}\right)}^{n-1} \leq \begin{cases}\pi\left\|D_{\tau} u\right\|_{L^{2}\left(\mathbb{S}^{n-1}\right)} & n=2 \\ 4\left\|D_{\tau} u\right\|_{L^{2}\left(\mathbb{S}^{n-1}\right)}^{2} \log \frac{8 e\left\|D_{\tau} u\right\|_{L^{\infty}\left(\mathbb{S}^{n-1}\right)}^{n-1}}{\left\|D_{\tau} u\right\|_{L^{2}\left(\mathbb{S}^{n-1}\right)}^{2}} & n=3 \\ C(n)\left\|D_{\tau} u\right\|_{L^{2}\left(\mathbb{S}^{n-1}\right)}^{2}\left\|D_{\tau} u\right\|_{L^{\infty}\left(\mathbb{S}^{n-1}\right)}^{n-3} & n \geq 4\end{cases}
$$

We now prove our result only for $n \geq 4$, since for $n=2,3$ the argument is the same. The above inequality and Lemma 2.7 lead to

$$
\|u\|_{L^{\infty}\left(\mathbb{S}^{n-1}\right)}^{n-1} \leq C(n)\left\|D_{\tau} u\right\|_{L^{2}\left(\mathbb{S}^{n-1}\right)}^{2}\|u\|_{L_{\left(\mathbb{S}^{n-1}\right)}^{\frac{n-3}{2}}},
$$

hence

$$
\|u\|_{L^{\infty}\left(\mathbb{S}^{n-1}\right)}^{\frac{n+1}{2}} \leq C(n)\left\|D_{\tau} u\right\|_{L^{2}\left(\mathbb{S}^{n-1}\right)}^{2}
$$

Since $\rho\|u\|_{L^{\infty}\left(\mathbb{S}^{n-1}\right)}=d_{\mathcal{H}}\left(\Omega, \Omega^{\#}\right)=\mathcal{A}_{\mathcal{H}}(\Omega)$, inequalities (3.16) and (3.14) give the result for $n \geq 4$.

Notice that, as expected, the final constant depends not only on the dimension, but also on the boundary parameter and on the size of the admissible sets for the maximization problem.

\section{FURTHER REMARKS AND OPEN PROBLEMS}

In the same spirit of [18], it is possible to prove the sharpness of the quantitative "weighted" isoperimetric inequality considered in the previous section for the functional $I(\cdot)$.

Theorem 4.1. Let $n \geq 2$. There exists a family of convex sets $\left(E_{\alpha}\right)_{\alpha}$ such that

$$
I\left(E_{\alpha}^{\#}\right)-I\left(E_{\alpha}\right) \rightarrow 0, \quad \text { when } \alpha \rightarrow 0
$$

and

$$
I\left(E_{\alpha}^{\#}\right)-I\left(E_{\alpha}\right) \simeq g\left(\mathcal{A}_{\mathcal{H}}\left(E_{\alpha}\right)\right)
$$

where $C$ is a suitable positive constant independent of $\alpha$.

We do not write the proof of this last theorem since, due to the analytic and non decreasing nature of the Bessel functions, it follows straightforward as in [18]. However, for the reader convenience, we explicitly recall the sets which give the sharpness of the exponents.

In the two dimensional case, to find the optimality of the exponent, one may use the "stadium" shaped sets (i.e. the convex hull of two balls with the same radius whose centers is connected by a segment passing through the origin) already considered in $[1,5]$. 
If $n \geq 4$, let $\alpha \in] 0, \pi / 2\left[\right.$ and $p_{\alpha} \in \mathbb{R}^{n}$ such that

$$
\left|p_{\alpha}\right|=\frac{1}{\cos \alpha}
$$

Then, we can define the set $E_{\alpha}$ as the convex hull of $B \cup\left\{-p_{\alpha}, p_{\alpha}\right\}$.

In the three dimensional case, the shape of the sets is quite different, due to the peculiar form of the function $g$. Let $\alpha \in] 0, \pi / 2\left[\right.$ and consider the following function $\omega_{\alpha}=\omega_{\alpha}(\varphi)$ defined over $\mathbb{S}^{2}$ and depending only on the spherical distance $\varphi$, with $\varphi \in[0, \pi]$, from a prescribed north pole $\xi^{*} \in \mathbb{S}^{2}$ :

$$
\omega_{\alpha}=\omega_{\alpha}(\varphi)= \begin{cases}-\sin ^{2} \alpha \log (\sin \alpha)+\sin \alpha(\sin \alpha-\sin \varphi) & \text { for } \sin \varphi \leq \sin \alpha \\ -\sin ^{2}(\alpha) \log (\sin \varphi) & \text { for } \sin \varphi \geq \sin \alpha\end{cases}
$$

Let $h_{\alpha}:=\omega_{\alpha}-\bar{\omega}_{\alpha}$, with $\bar{\omega}_{\alpha}$ the mean value of $\omega_{\alpha}$, i.e.

$$
\bar{\omega}_{\alpha}=\int_{0}^{\pi / 2} \omega_{\alpha}(\varphi) \sin \varphi d \varphi=(1-\log 2) \alpha^{2}+O\left(\alpha^{3}\right)
$$

when $\alpha$ goes to 0 , and let

$$
R_{\alpha}:=\left(1+3 h_{\alpha}\right)^{1 / 3}
$$

The $C^{1}$ function $R_{\alpha}=R_{\alpha}(\varphi)$ determines in polar coordinates $\left(R_{\alpha}, \varphi\right)$ a planar curve. By rotating this curve about the line $\xi^{*} \mathbb{R}$, we determine the boundary of a convex and bounded set depending on the parameter $\alpha$. We denote that set by $E_{\alpha}$.

Remark 4.2. The result of Theorem 4.1 suggests that sharpness of inequality (1.5) can be expected, but at the moment it seems hard to be proved.

An interesting question is whether the constraint on the perimeter is sufficient to ensure the ball to be the only solution for problem (1.3), even if the admissible sets are not necessarily convex (for instance, one can take the class of finite perimeter sets having the same perimeter). If one is able to prove in this more general framework the analogous of (1.4), in its quantitative version it should be suitable to replace the asymmetry function $\mathcal{A}_{\mathcal{H}}$ by some Fraenkel-type asymmetry function (for more details on the Fraenkel asymmetry, see [17]).

Acknowledgements. The first author would thank both Professor Nicola Fusco and the second author for the hospitality and the support during the research visit at the University Federico II of Naples that inspired this work. The second author was partially supported by the Academy of Finland grant 314227.

\section{REFERENCES}

[1] A. Alvino, V. Ferone and C. Nitsch, A sharp isoperimetric inequality in the plane. J. Eur. Math. Soc. 13 (2010) $185-206$.

[2] L. Ambrosio, N. Fusco and D. Pallara, Functions of bounded variation and free discontinuity problems. Oxford University Press, Oxford (2000).

[3] D.E. Amos, Computation of modified Bessel functions and their ratios. Math. Comput. 28 (1974) $239-251$.

[4] M. Bareket, On an isoperimetric inequality for the first eigenvalue of a boundary value problem. SIAM J. Math. Anal. 8 (1977) $280-287$.

[5] T. Bonnesen, Über das isoperimetrische Defizit ebener Figuren. Math. Ann. 91 (1924) 252-268.

[6] L. Brasco and G. De Philippis, 7 Spectral inequalities in quantitative form. In Shape optimization and spectral theory. Sciendo Migration (2017) 201-281.

[7] F. Brock and D. Daners, Conjecture concerning a Faber-Krahn inequality for Robin problems. Vol. 4 of Mini-Workshop: Shape Analysis for Eigenvalues (Organized by: D. Bucur, G. Buttazzo, A. Henrot), Oberwolfach Rep (2007) 1022-1023. 
[8] D. Bucur and G. Buttazzo, Vol. 65 of Variational methods in shape optimization problems. Springer-Progress in Nonlinear Differential Equations and Their Applications (2004).

[9] D. Bucur and S. Cito, Geometric control of the robin Laplacian eigenvalues: the case of negative boundary parameter. J. Geometr. Anal. 30 (2020) 4356-4385.

[10] D. Bucur, V. Ferone, C. Nitsch and C. Trombetti, A sharp estimate for the first Robin-Laplacian eigenvalue with negative boundary parameter. Preprint arXiv:1810.06108 (2018).

[11] D. Bucur, P. Freitas and J. Kennedy, Shape optimization and spectral theory, in The Robin Problem, edited by A. Henrot. De Gruyter Open, Warsaw (2017) 78-119.

[12] S. Cito, Existence and regularity of optimal convex shapes for functionals involving the robin eigenvalues. J. Convex Anal. 26 (2019) 925-943.

[13] L. Esposito, N. Fusco and C. Trombetti, A quantitative version of the isoperimetric inequality: the anisotropic case. Ann. Sc. Norm. Sup. Pisa Cl. Sci. 4 (2005) 619-651.

[14] V. Ferone, C. Nitsch and C. Trombetti, On a conjectured reverse Faber-Krahn inequality for a Steklov-type Laplacian eigenvalue. Commun. Pure Appl. Anal. 14 (2015) 63-82.

[15] P. Freitas and D. Krejčiřík, The first Robin eigenvalue with negative boundary parameter. Adv. Math. 280 (2015) $322-339$.

[16] B. Fuglede, Stability in the isoperimetric problem for convex or nearly spherical domains in $\mathbb{R}^{n}$. Trans. Am. Math. Soc. $\mathbf{3 1 4}$ (1989) 619-638.

[17] N. Fusco, The quantitative isoperimetric inequality and related topics. Bull. Math. Sci. 5 (2015) 517-607.

[18] N. Gavitone, D.A. La Manna, G. Paoli and L. Trani, A quantitative Weinstock inequality for convex sets. Calc. Var. Partial Differ. Equ. 59 (2020) 2.

[19] R. Schneider, Convex bodies: the Brunn-Minkowski theory. Number 151. Cambridge University Press (2014). 